\title{
Measurement Data Based Study on the Intra-body Propagation in the Presence of the Sternotomy Wires and Aortic Valve Implant
}

\author{
Mariella Särestöniemi, Carlos Pomalaza Raez, Timo Kumpuniemi, Matti Hämäläinen, Richard Kovacs, Jari Iinatti
}

\begin{abstract}
This paper presents a comprehensive study on the impact of sternotomy wires and a medical implant on the ultra-wideband (UWB) channel characteristics by studying intra-body propagation in the vicinity of on-body antennas. The main contribution on this work is to verify propagation path calculations with measurement data taken from a volunteer that has sternotomy wires and an aortic valve implant and from a reference volunteer without any implants. Furthermore, the impact of sternotomy wires and the aortic valve implant is confirmed by finite integration technique simulations using a tissue layer model. It is shown, that additional signal peaks and variations of the channel impulse responses are observed for the medical implant case, since both sternotomy wires and aortic valve implant contain highly conductive materials which impacts on the channel characteristics. In the time domain simulation results, the difference between the channel strength of the implanted and non-implanted cases varies between 5-17 dB within the time range corresponding to the relevant propagation paths. This phenomenon should be taken into account when designing on-body monitoring devices and when choosing sensor node locations to avoid interference due to the additional multipath propagated signal components. On the other hand, knowledge of the impact of the aortic valve implant may be utilized in the design of devices that can monitor the functionality of a valve implant during the heartbeat.
\end{abstract}

Index Terms-Aortic valve implant, intra-body propagation, channel characteristics, sternotomy wires, wireless body area networks.

\section{INTRODUCTION}

The study of the channel characteristics in wireless body area networks (WBAN) has been a very active area of research for several years. Numerous measurement campaigns and propagation simulations experiments have been conducted to gain a better understanding of the electromagnetic propagation in the vicinity of human tissues [1-25]. Recently, intra-body communications has become an intensive research topic. Several studies proposing intrabody channel characteristics, channel models, as well as new intrabody antennas have recently been published e.g. in [2-10].

Furthermore, the implant communication has become an important research topic [11-21]. Numerous studies have been presented on the radio channel characteristics between the transmitting implant and the on-body device, e.g in [11]. One of the main challenges in this field is the lack of the measurement data. Typically animals, or part of their organs, have been used to get the experimental knowledge about the intra-body propagation [11], [18-19].

Nowadays, computer simulators of electromagnetic (EM) waves propagation are common tools used to estimate the channel characteristics in the vicinity of human tissues [26-27]. Finite

Manuscript received March 8, 2018. This work has been funded by the project Wireless Body Area Networks Communications in the Congested Environments project at University of Oulu and in part Academy of Finland 6Genesis Flagship project (grant 318927).

M. Särestöniemi, T. Kumpuniemi, M. Hämäläinen, and J. Iinatti are with the Centre for Wireless Communications, University of Oulu, Finland. (phone: +358294482870, e-mail: firstname.surname@oulu.fi).
C. Pomalaza Raez is at Department of Electrical and Computer Engineering, Purdue University, Fort Wayne, Indiana, USA (e-mail:cpomalaz@purdue.edu R. Kovacs is at Indiana University Health Medical Center, Indiapolis, USA (email:rikovacs@iu.edu)

Integration Technique (FIT), Finite Element Method (FEM), Finite Difference Time Domain (FDTD) are all suitable tools for intra-body propagation prediction [28-30]. However, at some phase, it is also essential to verify the simulation based results with actual measurements.

Medical implants, such as pace makers and valve implants bring their own challenges to the study of propagation characteristics. Several of these implants contain steel, titanium alloys, tungsten, and other highly conductive materials and hence, have a strong impact on the channel characteristics in the vicinity where they are located. Furthermore, medical wires, staples, and bands, which are used for the closure after the operation [31], can also have significant impact since usually they are located close to the skin and hence near to the onbody antennas and sensor nodes.

Although the implant communications has been recently under an intensive study, the impact of the passive medical implants on the onbody radio channel characteristics is still a scarcely studied topic. The impact of a titanium based aortic valve implant on the ultra-wideband (UWB) channel characteristics was noticed for the first time in the measurement campaigns conducted for medical application purposes in [23]. The channel impulse responses (CIR) of a volunteer having an aortic valve implant had additional peaks due to the scattering from the aortic valve implant. Furthermore, the CIR was noted to drop off more quickly. In [24], some statistical parameters were presented to show the difference between the CIRs of different volunteers including the volunteer having an aortic valve implant. The main scope of [23] and [24] was to study UWB channel characteristics for medical applications - not just the impact of the aortic valve implant. These references did not include the impact of the sternotomy wires. The impact of the aortic valve was evaluated using a FEM-based 3-D simulator as shown in [25]. In the reference, new antenna locations were evaluated as well as the impact of the depth of the valve within the thorax on the CIR. The impact was minor with the antennas used and their locations for the case of a model of an adult having the valve at the depth of 7-8 cm, as well as with models of average adults as shown in Section II. If the valve was at the depth of $3 \mathrm{~cm}$, the impact was more significant.

The impact of an artificial aortic valve on the UWB on-body channel characteristics was studied in [32] by calculations, analysis of data measurements, and FIT -based power flow simulations. The paper describes the impact of the sternotomy wires on the channel characteristics as a novel topic in this field. The impact of the sternotomy wires on the channel characteristics was further studied in [33] using FIT-based simulations and a multi-layer tissue model. The presented results showed that sternotomy wires have clear impact on the channel characteristics. 
This paper further extends the studies presented in [32] and [33] by including detailed calculations for different intra-body propagation path options, new experimental measurements, and simulations results. The main contribution is to verify propagation path calculations with the experimental data and with simulation results for two cases, for a subject that has sternotomy wires and an aortic valve implant and for a subject without any implants

To our knowledge, this paper presents, for the first time, a comprehensive study on the impact of sternotomy wires and a medical implant on the channel characteristics by studying intra-body propagation in the vicinity of the on-body antennas. Previously published work have presented measurement data results, whereas this paper verifies the measurement data with intra-body propagation calculations and simulations taking account different propagation path options within the tissues. Furthermore, we present new measurement results with different antennas and different antenna locations.

This paper is organized as follows: Section II presents the studied scenario by describing the sternotomy wires and aortic valve implant, antennas and their locations, volunteers, simulation details, as well as measurement setup. Numerical calculations for the propagation paths inside the human body tissues are presented in Section III. Measurement data is presented and verified with the numerical calculations in Section IV. Simulations results are presented in Section V. Conclusions are given in Section VI.

\section{STUDY CASE}

\section{A. Sternotomy wires and aortic valve implant}

It is common for medical implants to contain metal or other highly conductive materials. Thus, implants will have a strong impact on the radio channel characteristics within the body, especially if they are located close to on-body antennas. For example, sternotomy wires, staples, and bands, which are used for the sternum closure after an open heart operation [31] are expected to have a clear effect on the channel characteristics.

In this study, sternotomy wires [31] are used as the sternum closure method. A Medtronic aortic valve implant [34], consisting of a titanium torus ring surrounded by a Teflon material, is also considered. The $1 \mathrm{~mm}$ thick sternotomy wires are made from steel, and they were wrapped around the sternum as it was closed after the valve replacement operation. Fig. 1a illustrates the location of the wires in the sternum as well as the position of the aortic valve with respect to the wires. The picture of the valve is also shown in Fig. 1a. The Medtronic aortic valve consists of a titanium torus ring surrounded by a teflon material. Details of the Medtronic valve are given in [34]. The x-ray figure of the volunteer having an aortic valve implant and sternotomy wires is shown, with his permission, in Fig, 1b. It is noted that the valve implant for this case is around $7 \mathrm{~cm}$ from the body surface.

a)
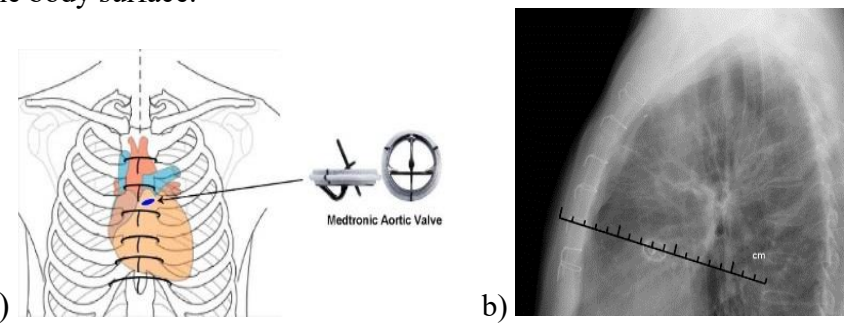

Figure 1. Location of the sternotomy wires and the aortic valve implant.

\section{B. Antennas and antenna locations}

For this study, we have used three different antennas designed for on-body and off-body communications: experimental loop and dipole [35], and a commercial SkyCross antenna [36]. The antennas were located on the chest with a $4 \mathrm{~mm}$ distance from the body surface. The antenna-body distance was selected to provide a reasonable operation mode for the antennas as well as keeping the distance as small as possible to be suitable for sensor nodes applications [35]. Two different antenna locations were studied: asymmetric and symmetric. The antenna separation distance in the asymmetric case was $6.5 \mathrm{~cm}$ and in the symmetric case $15 \mathrm{~cm}$. These separation distance, both suitable for medelical monitoring, were selected to study the impact of the sternotomy wires and aortic valve implant in both types of separation distances.

In the asymmetric case, the antennas were located asymmetrically with respect to the sternum. The transmitter antenna (Tx) was located on the left side of the chest and the receiver antenna (Rx) antenna on the middle of the sternum with a separation distance of $6.5 \mathrm{~cm}$. Figure 2 illustrates the locations of the antennas in the cross-section view of the human body. The distances between the Rx and Tx antennas and the aortic valve implant are approximately $7 \mathrm{~cm}$ and $9 \mathrm{~cm}$, respectively. Fig. 2 also shows the structure and dimensions of the wire loops as they are wrapped around the sternum. These loops are on the average $3 \mathrm{~cm}$ diameter and the wire itself is $1 \mathrm{~cm}$ of average thickness. Since the wires are $1 \mathrm{~mm}$ thick, the twisted ends are about $2 \mathrm{~mm}$ thick. The length of twisted end pair is approximately $2 \mathrm{~cm}$.

Fig. 3 presents the cross section view for the symmetric case where the distance between the antennas is $15 \mathrm{~cm}$. In this case, the distance between an antenna and the heart implant is approximately $9 \mathrm{~cm}$.

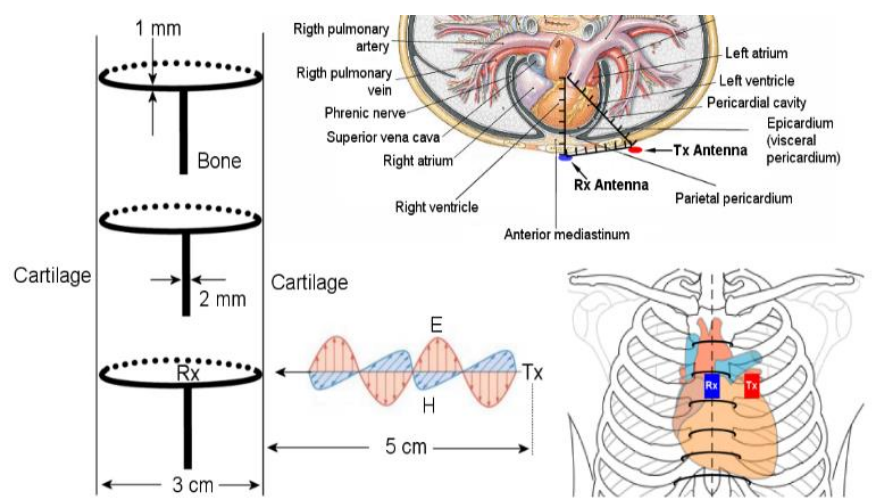

Figure 2. Antenna locations on the cross-section of the human body in the asymmetric case with antenna separation distance $6.5 \mathrm{~cm}$.

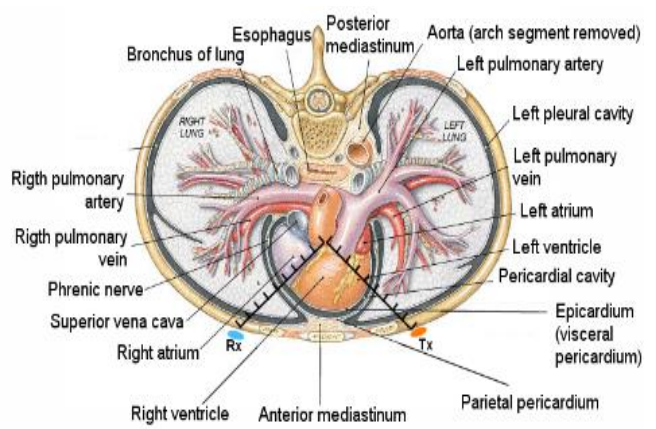

Figure 3. Antenna locations on the cross-section of the human body in the symmetric case with antenna separation distance $15 \mathrm{~cm}$.

\section{Volunteers}


Three male volunteers participated in the measurements. The metrics and age of the volunteers are summarized in the Table I. Volunteer A has a titanium alloy based aortic valve implant which was illustrated in Fig. 1. This volunteer has also steel sternotomy wires in his sternum, which were used for the sternum closure after the valve replacement. There are altogether six loop wires with the separation distance of approximately $2.5 \mathrm{~cm}$, as seen for x-ray presented in Fig1b.

Table I. Sizes of the volunteers.

\begin{tabular}{|l|l|l|l|}
\hline & A (with implant) & B (no implant) & C (no implant) \\
\hline Height & $170 \mathrm{~cm}$ & $181 \mathrm{~cm}$ & $173 \mathrm{~cm}$ \\
\hline Weight & $62 \mathrm{~kg}$ & $76 \mathrm{~kg}$ & $75 \mathrm{~kg}$ \\
\hline Age & 65 & 22 & 27 \\
\hline
\end{tabular}

\section{Simulations}

Simulations were conducted with the CST MicroWaveStudio software (CST) [38], which is a well-known EM-propagation simulator that uses the finite integration technique. The basic idea of FIT is to predict radio propagation by solving the Maxwell's equations in their integral form. Due to lack of the space, the corresponding Maxwell's equations are not repeated here but they can be found e.g. in [28] and [38].

A layered human body tissue model of the chest area is used to evaluate the channel characteristics in the vicinity and inside the human body. The layer model, illustrated in Fig. 4a, consists of the following tissues: skin, fat, muscle, bone, cartilage, heart, blood vessel, lung, and anterior mediastinum (ant. med). For simplicity sake, the tissues are modeled as rectangular blocks. The size of the whole layer model cube is $22 \mathrm{~cm} \times 13 \mathrm{~cm} \times 12 \mathrm{~cm}$. The thicknesses of the tissues are designed according to [12], as well as according to the $\mathrm{x}$ ray figure of the volunteer, with the aortic implant [33]. The dielectric properties for the different tissues were found in [39]. A simplified version of the aortic valve implant was modeled according to the material and dimension information provided in [34] and using the CST material library. This library provides enough materials properties for the valve modeling. The valve model was inserted in the heart layer of the tissue layer model so that the distance between the skin and valve is $7 \mathrm{~cm}$, as in the Fig. 1b. Furthermore, sternotomy wires with a thickness of $1 \mathrm{~mm}$ were modeled of steel material, and wrapped around the sternum layer rectangular. This way, the dimensions and materials of both layer model and implants are close to their real counterparts. For the reference case, a model without sternotomy wires and valve implant was also simulated.

Next, the simulation parameters are briefly summarized. The simulations were conducted for the $0-8 \mathrm{GHz}$ frequency bandwidth, with 2135 equally distributed frequency samples. Then number of mesh cells for the implant and non-implant models were $2.8 * 10^{9}$ and $1.1 * 10^{9}$, respectively. Such a large amount of mesh cells require parallel processing of several cluster nodes. We had 13 cluster nodes with CPU type: Intel(R) Xeon(R) CPU E5-2640 v4. Despite of an efficient parallel processing, the simulation time for the implant model was 4 days.

When carrying out EM-propagation computer simulations, it is essential to determine the boundary conditions properly and to define suitable and appropriate radiation environment for the model. CST provides several boundary condition options. Boundary condition "Open", which extends the touching geometry virtually to infinity, was selected to faces $1-5$ in the layer model cube (sides and the bottom) to avoid unintentional propagation via the sides, as shown in Fig. 4c. On the face 6 of the layer model cube, i.e., above the skin layer and antennas, the boundary condition was set "Open add space," which is similar to the "open" boundary condition but there is some extra space with distance $d_{\mathrm{s}}$ added to enable to proper functionality of the antennas [38]. The parameter $d_{\mathrm{s}}$ can be determined as an absolute distance or as the fraction of the wavelength

$$
d_{s}=\frac{\lambda}{n}
$$

where $n$ can be determined suitable for the simulation model [35]. In CST the default value for $n=4$ which is appropriate to our case as well [38]. For the rest of the simulation parameters, the default settings provided by the simulator are appropriate for our simulations case. Due to lack of the space, they are not discussed more in detail in this section, but they can be found in [38].

The excitation signal, which was in this case is a pure sinusoid signal, is fed to the Tx antenna, whose location in the layer model is shown in Fig. 4. The simulator calculates and provides frequency domain results such as the reflection coefficients for Tx and Rx antennas, S11, and S22, respectively. Besides, the channel responses S12 and S21 were also calculated. The frequency domain channel response is further converted into time domain by using the inverse fast Fourier transform (IFFT) function in Matlab to obtain the impulse response. Furthermore, Matlab is used for calculating the propagation time for each of the various path options as well as the power loss for each path, as described in Section III.

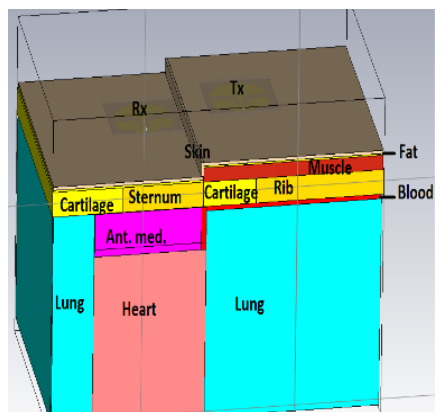

a)
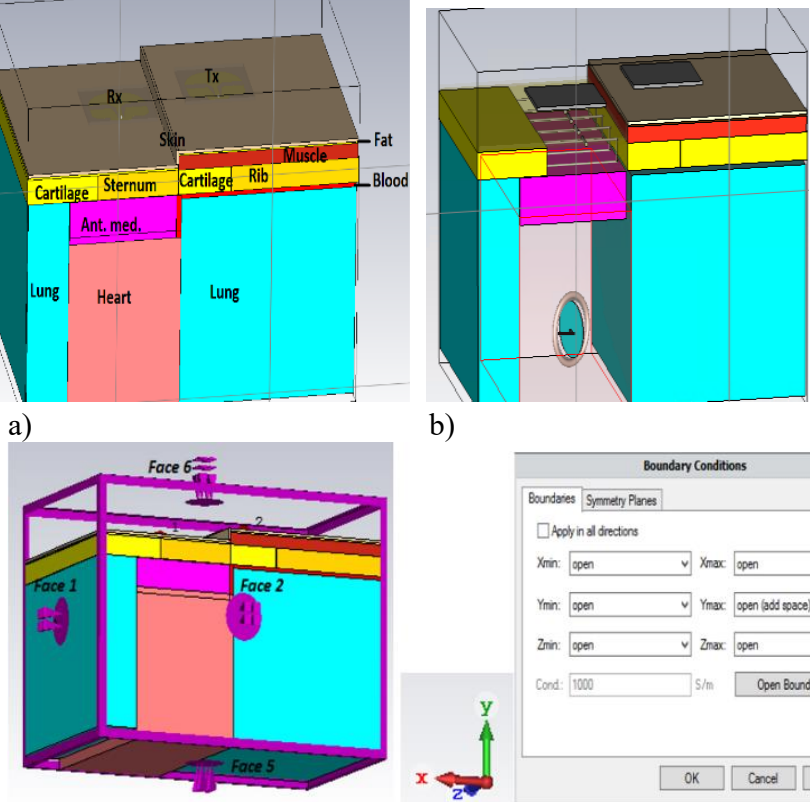

b)

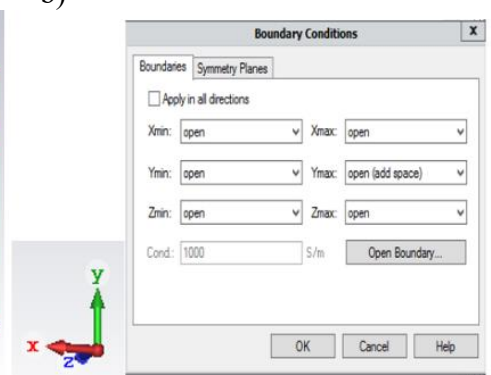

c)

Figure 4. Tissue layer model used in the simulations a) for the non-implanted case and b) for the implanted case, c) with boundary conditions selected.

\section{E. Measurements}

Two sets of measurements were conducted in an anechoic chamber. For the first set, a Vector Network Analyzer (VNA) Rohde \& Schwarz ZVA8 was used to measure within the $2-8 \mathrm{GHz}$ frequency band. For the second set, a VNA $8720 \mathrm{ES}$ was used to measure within the frequency band 3.1-10.6 GHz. These frequency bands were chosen since UWB is one of the physical layer options selected for IEEE802.15.6 standard and medical monitoring devices could be designed to work within that portion of the UWB band. We also selected large bandwidths to get good time resolution in the evaluations.

The antenna prototypes were connected to the VNA's ports using 8 $\mathrm{m}$ long Huber + Suhner SUCOFLEX 104PEA measurement cables. A proper calibration was performed before the measurements. The VNA was set to sweep 100 times for each scenario, collecting 1601 
frequency points for each measurement set. In the first measurement set, the frequency band was $2-8 \mathrm{GHz}$ and the antenna distance was $d_{\mathrm{A}}=6.5 \mathrm{~cm}$. Volunteers A (with implant) and B (without implant) participated in this measurement. In the second measurement set, volunteers $\mathrm{A}$ (with implant) and $\mathrm{C}$ (without implant) participated, the frequency band was $3.1-10.6 \mathrm{GHz}$ and $d_{\mathrm{A}}=15 \mathrm{~cm}$. In order to get the desired antenna-body distance, a $4 \mathrm{~mm}$ rohacell piece was set between the antenna and the human body.

The measurements were conducted in the frequency domain to obtain radio channel's frequency responses (S21 parameters), which were later transformed into time domain in Matlab using IFFT. Since the channel data is stored in the frequency domain, it will be possible to study impulse responses for different bandwidths and frequency ranges as the IFFT can be performed to the selected frequency band.

\section{NUMERICAL CALCULATIONS}

In this section, different path options for signal propagation within the human body tissues are introduced. In addition, numerical calculations for the propagation delays inside the human body are presented. In Subsection A we consider the asymmetric case with antenna separation distance of $6.5 \mathrm{~cm}$ and in Subsection B we consider the symmetric case with antenna separation distance of $15 \mathrm{~cm}$.

\section{A. Asymmetric case with antenna distance $6.5 \mathrm{~cm}$}

Fig. 2 in Section II illustrates the location of the antennas in a crosssection view of the human body. The distances between the antennas and the aortic valve implant are approximately $7 \mathrm{~cm}$ and $9 \mathrm{~cm}$, respectively. Next, we discuss the propagation path options from $\mathrm{Tx}$ to $\mathrm{Rx}$ antenna in the asymmetric case.

The dielectric properties of human tissues have significant impact on how the EM signal propagates. These dielectric properties vary significantly with the frequency and the tissue type. Dielectric values for different human tissues can be found in [39]. When considering signal propagation in the asymmetric case, one option is to consider the most direct path from the Tx antenna towards the valve/wire and further towards the Rx antenna, as presented in [32]. Another option is to consider, which of the possible paths are the fastest for propagation, i.e. for which the calculated propagation time is the smallest. When determining the propagation time, we first calculate the propagation velocity as,

$$
v=f \lambda
$$

where $f$ is the frequency and $\lambda$ is the wavelength in the tissue. Table II [39] summarizes the $\lambda$ ' $\mathrm{s}$ at the frequencies $2.5 \mathrm{GHz}, 3.5 \mathrm{GHz}, 5 \mathrm{GHz}$ and $8 \mathrm{GHz}$ for the tissues that are used in the propagation path options in this asymmetric case. Once we know the propagation velocity for each tissue, we can then calculate the propagation time $t_{d}$ taking account the distance $d$ that the signal travels in that tissue as

$$
t_{d}=\frac{d}{v}=\frac{d}{f \lambda} .
$$

Table II. Wavelengths for different tissues [39]

\begin{tabular}{|l|l|}
\hline Tissue & $\begin{array}{l}\text { Wavelength [m] } \\
2.5 \mathrm{GHz}, 3.5 \mathrm{GHz}, 5 \mathrm{GHz}, 8 \mathrm{GHz}\end{array}$ \\
\hline Air & $0.12,0.086,0.060,0.037$ \\
\hline Aorta & $0.018,0.012,0.009,0.006$ \\
\hline Blood & $0.015,0.010,0.010,0.005$ \\
\hline Bone Marrow (sternum) & $0.052,0.036,0.03,0.02$ \\
\hline Cartilage & $0.019,0.012,0.010,0.007$ \\
\hline Heart & $0.016,0.098,0.008,0.0054$ \\
\hline Lung Deflated & $0.017,0.011,0.0088,0.0057$ \\
\hline Lung Inflated & $0.026,0.016,0.013,0.0088$ \\
\hline Muscle & $0.016,0.011,0.008,0.0012$ \\
\hline Skin dry & $0.019,0.012,0.0099,0.0064$ \\
\hline
\end{tabular}

\section{Fat} $0.052,0.044,0.027,0.017$

Next, we will describe propagation path options for the case of asymmetric antennas with separation distance $6.5 \mathrm{~cm}$. Furthermore, we will calculate propagation time for each of these propagation path options.

Path 1: Skin: In this path option, the propagation is assumed to travel only through the skin, i.e., antenna distance of $6.5 \mathrm{~cm}$. Hence, the propagation time is at $2.5 \mathrm{GHz}$

$$
t_{T_{X} R x}=\frac{1}{f}\left(\frac{6.5 \cdot 10^{-2} \mathrm{~m}}{19 \cdot 10^{-3} \mathrm{~m}}\right)=1.36 \mathrm{~ns} .
$$

If there are medical wires in the sternum, part of the signal travels along the wires, as explained with details in [32-33]. The propagation speed is significantly higher in the wires and thus the propagation time is negligible compared to the propagation time in the tissues. For that case, the signal travels $5 \mathrm{~cm}$ in the skin until it reaches the wire, along which it travels until reaching the area of antenna, and from wire back on the skin surface and the antenna. In this case, the propagation time is $1.36 \mathrm{~ns}$ at $2.5 \mathrm{GHz}$. Similarly, the propagation times are calculated for the frequencies $3.5 \mathrm{GHz}, 5 \mathrm{GHz}$, and $8 \mathrm{GHz}$, which are summarized in Table III.

In the tables, the corresponding propagation path option through the wires are denoted as "_w". For instance, Path 1 describes the propagation path in the case of no wires/implant (reference case) and

\begin{tabular}{|c|c|}
\hline Tissue & $\begin{array}{l}\text { Wavelength [m] } \\
2.5 \mathrm{GHz}, 3.5 \mathrm{GHz}, 5 \mathrm{GHz}, 8 \mathrm{GHz}\end{array}$ \\
\hline Air & $0.12, \quad 0.086,0.060,0.037$ \\
\hline Aorta & $0.018,0.012,0.009,0.006$ \\
\hline Blood & $0.015,0.010,0.010,0.005$ \\
\hline Bone Marrow (sternum) & $0.052,0.036,0.03,0.02$ \\
\hline Cartilage & $0.019,0.012,0.010,0.007$ \\
\hline Heart & $0.016,0.098,0.008,0.0054$ \\
\hline Lung Deflated & $0.017,0.011,0.0088,0.0057$ \\
\hline Lung Inflated & $0.026,0.016,0.013,0.0088$ \\
\hline Muscle & $0.016,0.011,0.008,0.0012$ \\
\hline Skin dry & $0.019,0.012,0.0099,0.0064$ \\
\hline Fat & $0.052,0.044,0.027,0.017$ \\
\hline
\end{tabular}
the Path1_w describes the path option where there are sternotomy wires in the sternum (implanted case).

The Table III presents two propagation time values separated by slash (/) for each path option. The latter value is presented to ease the comparison between the calculated and measured results later in Section IV, since the value includes the delay of $0.3 \mathrm{~ns}$ due to cabling etc caused by the measurement setup. These values are presented in this section to avoid necessity to repeat the Table III in Section IV.

Furthermore, one should note that the implanted case has both propagation options, i.e., the part of the signal propagates through normal propagation path options (Path $\mathrm{X}$ ) and part through the wire option (Path X_w). Due to these additional propagation paths, the implanted case has usually more peaks visible in the impulse response.

Path 2: Skin-sternum-skin: In this propagation option, the signal travels about $5 \mathrm{~cm}$ through the cartilage or the skin (which have almost same dielectric properties) until it hits the sternum. The propagation time for signal travelling $5 \mathrm{~cm}$ in the skin or the cartilage is

$$
t_{T_{X} \text { Sternum }}=\frac{1}{f}\left(\frac{5.0 \cdot 10^{-2} \mathrm{~m}}{19 \cdot 10^{-3} \mathrm{~m}}\right)=\frac{1}{2.5 \cdot 10^{9} \mathrm{~Hz}} \frac{50}{19}=1.05 \mathrm{~ns}
$$


The average width of the sternum is about $3 \mathrm{~cm}$ with a thickness of about $1 \mathrm{~cm}$. The antenna is centered on the sternum. The EM travels through the sternum towards the antenna for about $1.5 \mathrm{~cm}$, and then passes through the fat and skin tissues reaching the Rx. The thickness of the fat and skin tissues on the sternum area assumed to be $0.6 \mathrm{~cm}$ and $0.15 \mathrm{~cm}$ (lean person), respectively according to [9]. Hence, the propagation time is calculated at $2.5 \mathrm{GHz}$

$$
\begin{gathered}
t_{\text {Ster }-R x}=\frac{1}{f}\left(\frac{1.5 \cdot 10^{-2} \mathrm{~m}}{52 \cdot 10^{-3} \mathrm{~m}}\right)+\frac{1}{f}\left(\frac{1.5 \cdot 10^{-3} \mathrm{~m}}{19 \cdot 10^{-3} \mathrm{~m}}\right)+\frac{1}{f}\left(\frac{6 \cdot 10^{-3} \mathrm{~m}}{52 \cdot 10^{-3} \mathrm{~m}}\right) \\
=0.19 \mathrm{~ns} .
\end{gathered}
$$

The propagation time from $\mathrm{Tx}$ antenna to $\mathrm{Rx}$ antenna via the cartilage and sternum is then (using the values for $2.5 \mathrm{GHz}$ )

$$
t_{T x \text {-cartilage-sternum-Rx }}=1.05 \mathrm{~ns}+0.19 \mathrm{~ns}=1.24 \mathrm{~ns} .
$$

In the presence of sternotomy wires, the part of the signal may travel through the wires. Hence, the propagation time is calculated taking into account the total distance in the skin $(5.15 \mathrm{~cm})$ and fat $(0.6 \mathrm{~cm})$ at $2.5 \mathrm{GHz}$ as

$$
t_{T x-R x}=\frac{1}{f}\left(\frac{5.15 \cdot 10^{-2} \mathrm{~m}}{52 \cdot 10^{-3} \mathrm{~m}}\right)+\frac{1}{f}\left(\frac{6 \cdot 10^{-3} \mathrm{~m}}{52 \cdot 10^{-3} \mathrm{~m}}\right)=1.1 \mathrm{~ns} .
$$

Similarly, the propagation time is calculated for the frequencies 3.5 $\mathrm{GHz}, 5 \mathrm{GHz}$ and $8 \mathrm{GHz}$, which are summarized in Table III.

Path 3 Skin-fat-skin: In this path option, the signal travels through the skin $(0.15 \mathrm{~cm})$ layer reaching the fat layer, along which the signal travels about $6.5 \mathrm{~cm}$. Then it travels through the skin layer again $(0.15$ $\mathrm{cm}$ ) and reaches the Rx. In the presence of the sternotomy wires, part of the signal propagates through the wires, which decreases the propagation time. In this case, the propagation time is around $0.52 \mathrm{~ns}$ and in the presence of wires only $0.44 \mathrm{~ns}$. . As discussed in [5], fat is a good propagation channel for intra-body communications.

Table III

Propagation times [ns] for asymmetric case with antenna distance $6.5 \mathrm{~cm}$.

\begin{tabular}{|l|l|l|l|l|}
\hline Frequency & $2.5 \mathrm{GHz}$ & $3.5 \mathrm{GHz}$ & $5 \mathrm{GHz}$ & $8 \mathrm{GHz}$ \\
\hline Path 1 & $1.34 / 1.64$ & $1.33 / 1.63$ & $1.31 / 1.61$ & $1.27 / 1.57$ \\
\hline Path 1_w & $1.08 / 1.38$ & $1.06 / 1.36$ & $1.04 / 1.34$ & $1.02 / 1.32$ \\
\hline Path 2 & $1.27 / 1.57$ & $1.23 / 1.53$ & $1.22 / 1.52$ & $1.11 / 1.41$ \\
\hline Path2w & $1.11 / 1.41$ & $1.11 / 1.41$ & $1.09 / 1.39$ & $1.05 / 1.35$ \\
\hline Path 3 & $0.56 / 0.86$ & $0.55 / 0.85$ & $0.54 / 0.84$ & $0.53 / 0.83$ \\
\hline Path3_w & $0.45 / 0.75$ & $0.44 / 0.74$ & $0.43 / 0.73$ & $0.42 / 0.72$ \\
\hline Path 4 & $1.39 / 1.69$ & $1.37 / 1.67$ & $1.35 / 1.65$ & $1.30 / 1.60$ \\
\hline Path 4w & $1.37 / 1.67$ & $1.36 / 1.66$ & $1.33 / 1.63$ & $1.29 / 1.59$ \\
\hline Path 5_D & $1.56 / 1.86$ & $1.56 / 1.86$ & $1.51 / 1.81$ & $1.45 / 1.75$ \\
Path 5_I & $1.32 / 1.62$ & $1.30 / 1.70$ & $1.30 / 1.70$ & $1.23 / 1.53$ \\
\hline Path5w_D & $1.49 / 1.79$ & $1.49 / 1.79$ & $1.43 / 1.73$ & $1.38 / 1.78$ \\
Path5w_I & $1.24 / 1.54$ & $1.22 / 1.52$ & $1.21 / 1.51$ & $1.16 / 1.46$ \\
\hline Path 6_D & $3.03 / 3.33$ & $2.97 / 3.27$ & $2.92 / 3.32$ & $2.81 / 3.11$ \\
\hline Path 6_I & $2.87 / 3.17$ & $2.83 / 3.13$ & $2.76 / 3.06$ & $2.65 / 2.95$ \\
\hline Path6w_D & $2.96 / 3.26$ & $2.89 / 3.19$ & $2.85 / 3.15$ & $2.73 / 3.03$ \\
Path6w_I & $2.79 / 3.09$ & $2.75 / 3.05$ & $2.69 / 2.99$ & $2.58 / 2.88$ \\
\hline
\end{tabular}

Path 4 Skin-fat-muscle-bone/wire-fat-skin: In this path option, the signal mainly travels through the ribs $(5 \mathrm{~cm})$. Naturally, the signal has to pass through the skin $(0.15 \mathrm{~cm})$, fat $(0.6 \mathrm{~cm})$, and muscle $(0.5 \mathrm{~cm})$ layers before reaching the bones. The propagation time would be 1.37 ns at $3.5 \mathrm{GHz}$. In the presence of the wires, the propagation time would be $1.35-1.45 \mathrm{~ns}$ in the measured frequency band.

Path 5 Skin-fat-muscle-lung-anterior mediastinum-bone/wire-fatskin: This propagation path option considers the main propagation through the lungs from $\mathrm{Tx}$ to $\mathrm{Rx}$ antenna. The distance travelled through the lungs is approximately $3 \mathrm{~cm}$. After passing the lungs, the signal travel through the anterior mediastinum $(1 \mathrm{~cm})$ reaching the sternum. The propagation time is $2.01-2.39 \mathrm{~ns}$ depending if the lungs are inflated (I) or deflated (D). These abbreviations are used in the tables to clarify the propagation time as the lungs are inflated (Path5_I) or deflated (Path5_D). Also in this case, the signal passing through skin, fat, and muscle layers is also taken into account. In the presence of the wires, part of the signal travel via the wires whereas without the wires it will go through the sternum, fat and skin tissue before reaching the Rx antenna. Hence, the propagation distance without the wire option is $1.17-2.21 \mathrm{~ns}$.

Path 6 Skin-fat-muscle-lung-heart-valve-heart-anterior mediastinum-bone/wire-fat-skin: In [23]-[24], [32] it has been shown that titanium alloy based aortic valve implant has an impact on the channel characteristics. Here we consider the propagation path, which reflects from the valve.

The most direct path from Tx to the valve travels through the skin, fat, muscle, bone, lung $(2 \mathrm{~cm})$ and heart $(4 \mathrm{~cm})$. The reflected signal goes back through the heart $(4 \mathrm{~cm})$, anterior mediastinum $(1 \mathrm{~cm})$, fat, and skin. Only the dimensions of the tissues, which differ from the previous cases, are marked. The propagation time, which is calculated similarly to the previous cases, is $3.38 \mathrm{~ns}$ in the case of deflated lungs and $3.20 \mathrm{~ns}$ in the case of inflated lungs. These both values are calculated just to emphasize the impact of the respiration on the propagation delays. Part of the signal may travel via the sternotomy wires instead of the sternum, which enhances the propagation. In that case the propagation time with the deflated and inflated lungs is 3.15 ns and $2.98 \mathrm{~ns}$, respectively.

It should be noted that reflections from the medical wires may occur also from the wires above or below the antenna - not just in the nearest wires. The signal can travel along the wires horizontally but also vertically along the twisted ends of the wires. For instance, as the signal travels through muscles, it can propagate until the medical wire which is $2.5 \mathrm{~cm}$ below the antenna. From the wire it can continue upwards towards the Rx antenna so that it needs to travel via the sternum bone only $1.5 \mathrm{~cm}$ before reaching the Rx. In this case, the propagation time is only between $1.79-1.92 \mathrm{~ns}$ for the measured band. There is a reflection from the sternotomy wire which is $5 \mathrm{~cm}$ below the antenna, the corresponding propagation times is $2.0-2.2 \mathrm{~ns}$. All these possible propagation paths can be seen as variation between the CIR samples. Thus, the variation between the samples is stronger with the implant case than with the non-implant case.

\section{A. Symmetric case with antenna distance $15 \mathrm{~cm}$}

In the symmetric case, the $\mathrm{Rx}$ and $\mathrm{Tx}$ antennas are located on the chest symmetrically respect to the sternum as shown in Fig. 5 providing more propagation options. The propagation path options are described in the following.

Path 1 a) Skin-fat-muscle-cartilage-sternum/wires-muscle-fat-skin and b) Skin-sternum/wires-fat-skin. The propagation path option 1a consists of the route from Tx to Rx through the skin $(0.15 \mathrm{~cm})$, fat $(0.6$ $\mathrm{cm})$, muscle $(0.5 \mathrm{~cm})$, cartilage $(3 \mathrm{~cm})$, sternum $(3 \mathrm{~cm})$, cartilage $(3$ $\mathrm{cm})$, muscle, fat and skin. The propagation velocities through the skin and through the cartilage are about the same and thus the propagation time for these path options are almost the same. The only difference comes from the fat tissue calculated only once in the case (a), whereas it needs to be multiplied by two if the signal goes until the cartilage tissue case (b). For the case (a), propagation times for the chosen bandwidth is $2.59-2.75 \mathrm{~ns}$. For the case (b), it is $2.65-2.82 \mathrm{~ns}$. In the presence of sternotomy wires, the signal may propagate via the wires instead of the sternum bone. In this case, the propagation times for the cases (a) and (b) are $2.37-2.53 \mathrm{~ns}$ and $2.43-2.60 \mathrm{~ns}$, respectively.

Path 2: Skin-fat-muscle-bone-cartilage-sternum-cartilage-bonemuscle-fat-skin: In Path 2, the signal travels though the rib $(4 \mathrm{~cm}$ bone), cartilage $(2 \mathrm{~cm})$, sternum $(3 \mathrm{~cm}$ bone), cartilage $(2 \mathrm{~cm})$, and 
again the rib (4 $\mathrm{cm}$ bone). Since the signal travels through the skin, fat, and muscle tissues, their impact is also included in the total propagation time. The total propagation time will be $2.07-2.19 \mathrm{~ns}$ in the considered frequency band. As in the previous case, in the presence of the sternotomy wires, the signal may travel partly though the wires. In this case total propagation time is $1.28-1.40 \mathrm{~ns}$.

Path 3: Rib (4 cm bone), skin (3+ cm), sternum (3 cm bone), skin $(3+\mathrm{cm}), \mathrm{rib}(4 \mathrm{~cm}$ bone $)$. Since the propagation velocity within the skin is about the same as within the cartilage, the overall propagation time for waves taking this path is about the same as for those in Path 2. Similarly as in the case of Path 2, the medical wires may diminish the propagation time if part of the signal travels through the wires in the sternum.

Path 4: Skin-fat-skin: In this propagation path option, the signal travels only through the fat layer $(15 \mathrm{~cm})$. Propagation time is surprisingly small, only roughly $1.0 \mathrm{~ns}$. The presence of wires further diminishes the propagation time.

Path 5: Skin-fat-muscle-lung-anterior mediastinum-bone/wire-fatskin: This propagation path option considers the propagation through the lungs from Tx to Rx antenna. The distance travelled through the lungs is approximately $2.5 \mathrm{~cm} /$ side, i.e., altogether $5.0 \mathrm{~cm}$. After passing the lungs, the signal travels through anterior mediastinum (1 $\mathrm{cm}$ ) reaching the sternum and continues further through the anterior mediastinum and lungs on the right side of the chest. Then it propagates further until Rx antenna via muscle, fat and skin tissues. The propagation time is $1.55-2.12 \mathrm{~ns}$ depending on if the lungs are inflated or deflated. In the presence of the wires, part of the signal travels via the wires whereas without the wires it will go through the sternum, fat and skin tissue before reaching the Rx antenna. Hence, the propagation distance without the wire option is $1.33-1.56 \mathrm{~ns}$.

Path 6: Skin-fat-muscle-fat-skin: This option considers that signal travels through the muscles, which are between the ribs. In this case, the total propagation time consists of the time that takes to travel through skin $(0.2 \mathrm{~cm})$, fat $(0.9 \mathrm{~cm})$, muscle $(6 \mathrm{~cm})$, sternum $(3 \mathrm{~cm})$, muscle $(6 \mathrm{~cm})$, fat $(0.9 \mathrm{~cm})$ and skin $(0.2 \mathrm{~cm})$, resulting total propagation time 3.13 - $3.32 \mathrm{~ns}$. In the presence of sternotomy wires, by which the signal can propagate instead of the sternum bone, the total propagation time would be $2.91-3.09 \mathrm{~ns}$.

Path 7: This propagation path option considers possible reflection from the aortic valve implant through the most direct way from the Tx antenna to the valve. As shown in Fig. 3, the signal would go through the skin, fat, muscle, lung $(2.5 \mathrm{~cm})$, and heart $(4 \mathrm{~cm})$, where it hits the titanium based ring of the valve structure and the part of the signal reflects towards Rx antenna through the heart, lungs, muscle, fat, and skin. The propagation time within the studied bandwidth is $3.33-3.58$ $\mathrm{ns}$ in the case on deflated lungs and $2.92-3.17 \mathrm{~ns}$ with the inflated lungs.

Path 8: This propagation path option considers the possible reflections from the aortic valve implant. In this case, a signal of the Tx-valve link is propagated through the aorta, since propagation velocity is relatively high in the blood and blood vessels. The valve$\mathrm{Rx}$ link is calculated similarly as in the previous path option since on the right side of the chest, the blood vessel does not reach the vicinity of the Rx-antenna and hence, the direct path is faster. Thus, the propagation path consists of skin, fat, muscle, lung $(2 \mathrm{~cm})$, blood $(10.5$ $\mathrm{cm})$, valve, heart $(4 \mathrm{~cm})$, lung $(2 \mathrm{~cm})$, muscle, fat, and skin. The total propagation times result to $3.41-3.65 \mathrm{~ns}$ in the case on deflated lungs and $3.1-3.34 \mathrm{~ns}$ in the case on inflated lung.

In all intra-body paths, as the EM traverse the sternum they will interact with the sternotomy wires. This interaction will cause dispersion of the waves in several directions but also the wire array will store and release energy across time, i.e. like a solenoid being impacted by an EM wave. All this can be seen as a strong variation in the impulse responses.

\section{Measurement Results}

In this section, several measurement results are presented and compared to the propagation path calculations in order to verify the impact of the sternotomy wires and the aortic valve implant. First, we

evaluated the delay due to the measurement setup (antennas, cabling, etc) by measuring the antennas at free-space in the anechoic chamber and comparing it with the free-space propagation calculation. For the case of the $15 \mathrm{~cm}$ separation, the line-of-sight (LOS) in the air propagation time from $T_{X}$ to $R_{X}$ is

$$
t_{T_{X} R_{X}}(L O S)=\frac{1}{f}\left(\frac{15 \cdot 10^{-2} \mathrm{~m}}{86 \cdot 10^{-3} \mathrm{~m}}\right)=0.50 \mathrm{~ns} .
$$

When only the antennas (separated by $15 \mathrm{~cm}$ ) are in the anechoic chamber, the signal at the receiver is shown in Fig. 5. The first peak arrives about $0.80 \mathrm{~ns}$. The difference of $0.30 \mathrm{~ns}$ with the LOS epoch computed above is due to the delay introduced by the antenna system (including the cables) and the UWB pulse shape used [41]. This delay should be taken into account when discussing the calculations and results for other measurements described next and thus it was added for the propagation time values presented in Tables II and III in Section III.

Table IV

Propagation times [ns] in the symmetric with antenna distance $15 \mathrm{~cm}$.

\begin{tabular}{|l|l|l|l|l|}
\hline Path/frequency & $3,5 \mathrm{GHz}$ & $5 \mathrm{GHz}$ & $8 \mathrm{GHz}$ & $10 \mathrm{GHz}$ \\
\hline Path 1a & $2.75 / 3.05$ & $2.72 / 3.02$ & $2.67 / 2.97$ & $2.59 / 2.89$ \\
Path 1a_w & $2.44 / 2.74$ & $2.43 / 2.73$ & $2.37 / 2.77$ & $2.28 / 2.58$ \\
Path 1b & $2.82 / 3.12$ & $2.79 / 3.09$ & $2.73 / 3.03$ & $2.65 / 2.95$ \\
Path 1b_w & $2.60 / 2.90$ & $2.56 / 2.86$ & $2.51 / 2.81$ & $2.43 / 2.73$ \\
\hline Path 2\&3 & $2.19 / 2.49$ & $2.18 / 2.48$ & $2.13 / 2.43$ & $2.07 / 2.37$ \\
Path 2\&3w & $1.40 / 1.70$ & $1.34 / 1.74$ & $1.32 / 1.72$ & $1.28 / 1.58$ \\
\hline Path 4 & $1.22 / 1.52$ & $1.20 / 1.50$ & $1.18 / 1.48$ & $1.15 / 1.45$ \\
\hline Path 4w & $1.09 / 1.29$ & $1.08 / 1.38$ & $1.07 / 1.37$ & $1.04 / 1.34$ \\
\hline Path 5_D & $2.11 / 2.41$ & $2.12 / 2.42$ & $2.00 / 2.30$ & $1.93 / 2.23$ \\
Path 5_I & $1.67 / 1.97$ & $1.70 / 2.00$ & $1.60 / 1.90$ & $1.55 / 1.85$ \\
\hline Path5w_D & $1.89 / 2.19$ & $1.81 / 2.11$ & $1.74 / 2.04$ & $1.71 / 2.01$ \\
Path5w_I & $1.45 / 1.75$ & $1.45 / 1.75$ & $1.37 / 1.67$ & $1.33 / 1.63$ \\
\hline Path 6 & $3.32 / 3.52$ & $3.28 / 3.58$ & $3.19 / 3.49$ & $3.13 / 3.43$ \\
\hline Path6w & $3.09 / 3.39$ & $3.03 / 3.33$ & $2.97 / 3.27$ & $2.91 / 3.21$ \\
\hline Path 7_D & $3.58 / 3.88$ & $3.46 / 3.76$ & $3.45 / 3.75$ & $3.30 / 3.70$ \\
\hline Path 7_I & $3.17 / 3.47$ & $3.12 / 3.42$ & $3.05 / 3.35$ & $2.92 / 3.22$ \\
\hline Path 8_D & $3.68 / 3.98$ & $3.65 / 3.95$ & $3.51 / 3.81$ & $3.41 / 3.71$ \\
\hline Path 8_I & $3.33 / 3.53$ & $3.34 / 3.64$ & $3.21 / 3.51$ & $3.10 / 3.40$ \\
\hline
\end{tabular}

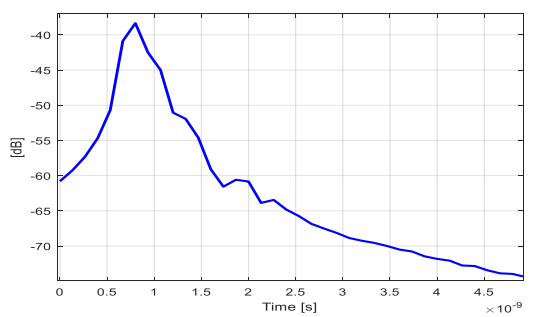

Figure 5. Impulse response of the loop antennas in the anechoic chamber with the antenna distance $15 \mathrm{~cm}$.

\section{A. Antenna separation distance $6.5 \mathrm{~cm}$}

In [29], we showed several impulse responses for this measurement scenario. In this paper, some of them are repeated for clarity since new analysis and discussion about the results are presented. 
First, we study the channel impulse responses with the SkyCross antenna having the separation distance is $6.5 \mathrm{~cm}$. Figs. 6a-b [32] present the comparison between the impulse responses with implanted and non-implanted volunteers, respectively.

For both cases, the main peak arrives at the same time, approximately $0.5 \mathrm{~ns}$. This is the line-of-sight propagation peak. At time instant $1 \mathrm{~ns}$ the second peak arrives for both cases, which is assumed to be the signal propagation via creeping waves. The main peak is quite wide it reaches up to $0.8 \mathrm{~ns}$. The reason for this is that the propagation signal which travels through the fat layer, arrives already at this time range, as calculated in Section III for Path 3.

There are variations in the timing and strength of the peaks for several reasons: the propagation time is different for different frequencies and also breathing has an impact. Besides, on the case of the volunteer with sternotomy wires and aortic valve implant, there are reflections from the several wires and also from the implant, which all cause variation in the channel responses, as discussed earlier.

When we study the channel impulse responses of the implanted and non-implanted cases in Figs 6a-b, we see that in both cases there are visible peaks after $1.6 \mathrm{~ns}$. These correspond to the propagation Path 1 described in Section III. Instead, the response strength is high around 1.3 ns only in the case of implanted volunteer, since for Path 1, the propagation is further enhanced if there are wires in the sternum. Also for these cases, propagation calculations for paths 2, 4, and 5 shows that there should be signals arriving around $1.6 \mathrm{~ns}$ for non-implant and around $1.2-1.3 \mathrm{~ns}$ for implanted cases. Thus, in the time slot $1.5-1.9$ $\mathrm{ns}$ there are variations between the CIR samples because according to the calculations, propagation time for several path options coincides in this time slot.

As discussed in Section III the reflections from the other loop wires will arrive around $1.79-1.92 \mathrm{~ns}$ ( $2.5 \mathrm{~cm}$ away from the antennas) and $2.0 \mathrm{~ns}-2.2 \mathrm{~ns}(5.0 \mathrm{~cm}$ away from the antennas). When the $0.30 \mathrm{~ns}$ delay is included, we will get $1.99-2.12 \mathrm{~ns}$ and $2.3-2.5 \mathrm{~ns}$. These peaks can also be seen in the impulse responses of the implanted volunteer's data.

Furthermore, peaks due to the reflection from the aortic valve (Path 6) can be seen from the time instant around $2.7-3.3 \mathrm{~ns}$. At that time instant, the channel is attenuated strongly, though still clearly visible. There are variations between the arriving time and strength depending on the respiration (signal travel partially through the lungs) and the path option.

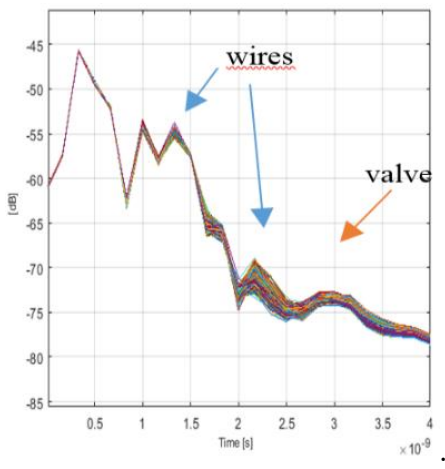

a)

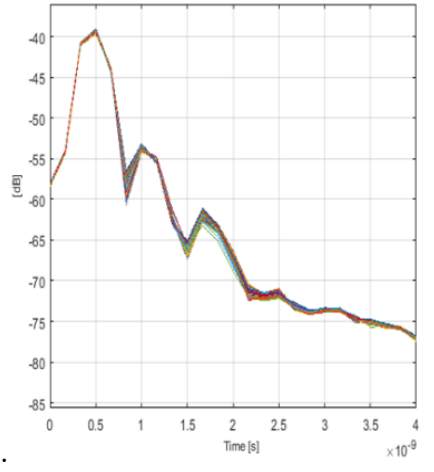

b)
Figure 6. Impulse responses with the SkyCross antenna for a) implanted and b) non-implanted volunteer's cases [32].

Next, we study the impulse responses with 3D plots and contours plots, which clearly illustrate/visualize the variations between the impulse response samples from both the implant and non-implant cases, in more details. Fig. 7 a-b illustrates the 3D plots and contour plots with SkyCross antenna for the implanted volunteer, respectively. Figs. 7c-d illustrates the 3D and contour plots with SkyCross antenna for the non-implanted volunteer, respectively. These 3D plots illustrate the attenuation profile of the channel and the rough variation between the CIR samples. The additional peak line due to the reflections from the sternotomy wires is evident. Besides, the peak line caused by the reflections from the aortic valve implant is noticeable. However, these differences can be seen more clearly in the contour plots presented below the 3D plots. Comparing the contour plots of the implanted and non-implanted volunteer's cases at the time instant of $1.3 \mathrm{~ns}$, we can note clear differences. For the non-implanted case, there is just a few contour lines whereas for the implanted case there are several lines representing steep changes in the impulse responses. Similarly, at the time instant of $2.5 \mathrm{~ns}$, which corresponds to the time instant for the reflections from the further loop wires, the difference between the contour plots of implanted and non-implanted cases is noticeably. For the non-implant case, there are almost no lines on that area whereas for the implant case, there are several contours lines whose density varies within the samples. Similarly, the reflections due to the valve around $3.0 \mathrm{~ns}$ is clearly seen.
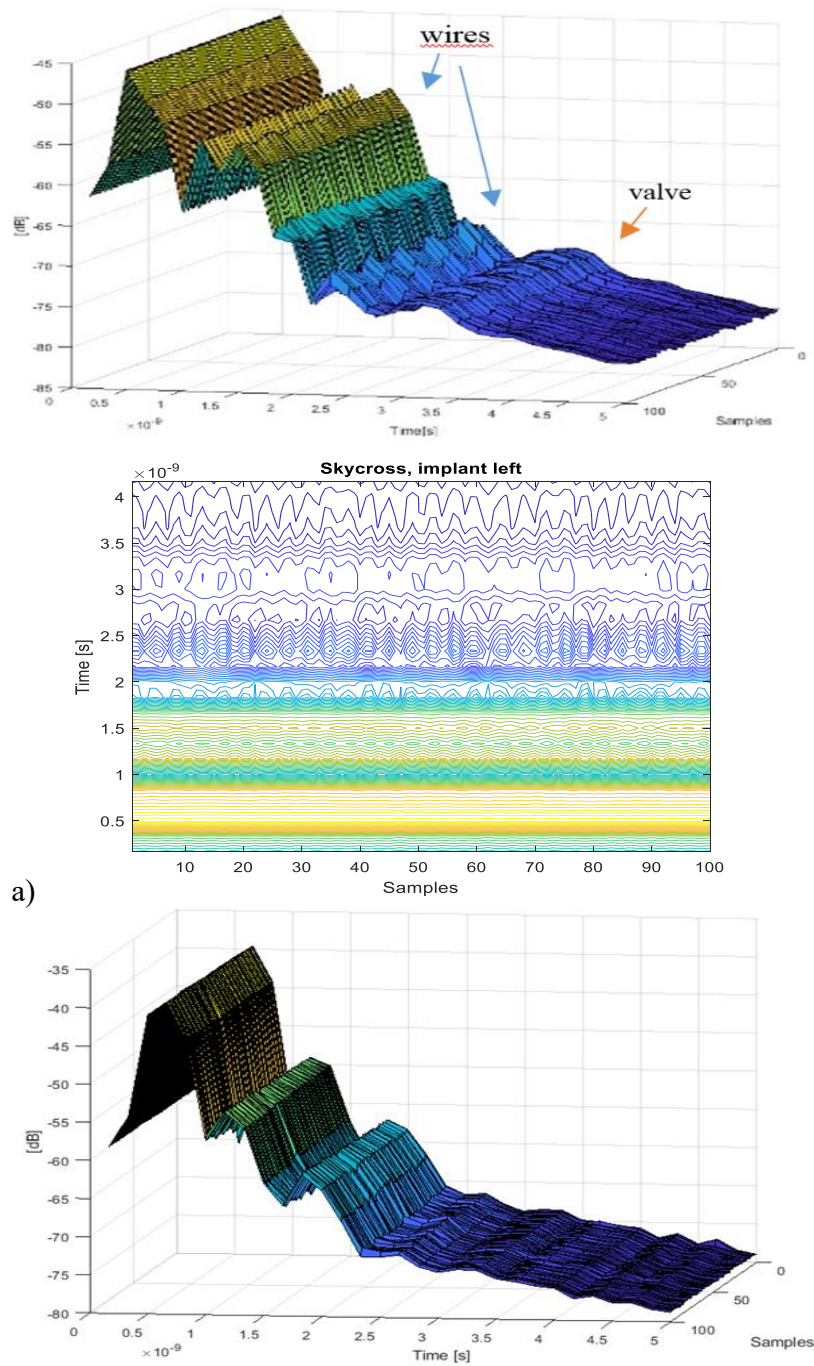


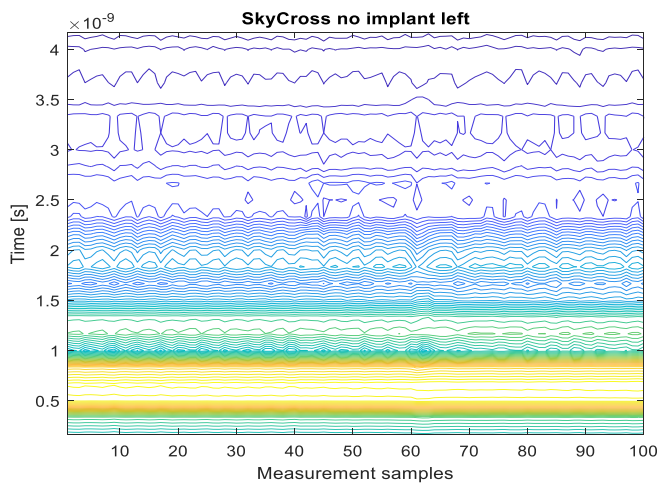

b)

Figure 7. 3D plot and contours of the impulse responses obtained with SkyCross antenna a) with implanted volunteer, b) non-implanted volunteer.

\section{B. Antenna separation distance $15 \mathrm{~cm}$}

Next, we will evaluate the impact of the medical wires and the aortic implant for the case with antenna separation distance $15 \mathrm{~cm}$. Figures 9-10 present all the impulse response samples obtained with the volunteer A (implanted) and with volunteer C (no implant), respectively. Since the variation between the impulse response samples is large, the mean over all the impulse responses is also plotted in the figures. For the implanted and non-implanted volunteer's case dash-dot black line and blue solid line are used, respectively. As it can be noted, especially in the case of the implanted volunteer, averaging operation diminishes some of the peaks.

For both cases, the main peak arrives at the same time, approximately at $0.8 \mathrm{~ns}$, which is the line-of-sight propagation peak. The main peak is followed by creeping waves as well as the fast propagation through the fat tissue (Path 4). The peaks due to the Path 4 and Path4_w can clearly be seen around $1.5 \mathrm{~ns}$ and $1.3 \mathrm{~ns}$, respectively.

According to the calculation presented for the symmetric case in Section III, waves via Path 1 should arrive at time instants $2.59-2.82$ ns. For this case, we need to add $0.30 \mathrm{~ns}$ due to the cabling delays, antennas setup, etc., resulting on instants $2,89 \mathrm{~ns}-3.12 \mathrm{~ns}$, including both cases a and $b$. The effect of this path option can be clearly seen in the impulses responses of the volunteers A (with implant) and B (without the implant) since there is an obvious peak in those epochs. There are variations in the attenuation and timing between the samples, especially in the CIR of the volunteer A. Furthermore, in the impulse responses of the implanted volunteer we can see strong variations at time instants $2.58-2.90 \mathrm{~ns}$ which correspond to the cases a) and b) as the signal propagates partly through the wires.

Furthermore, as described in the subsection about the antenna distance of $6.5 \mathrm{~cm}$, there are also reflections from further away sternotomy wires and the signal can also propagate through the wires vertically. For instance, the signal that passes along the wires that are $2.5 \mathrm{~cm}$ above/below the antennas, arrive at the receiver approximately 3.1-3.4 ns. According to the calculations presented in Section III, Path 2 and Path 3 should arrive approximately at the time instant $2.5 \mathrm{~ns}$ when the $0.30 \mathrm{~ns}$ delay is included. This is also clearly seen in all the impulse responses. In the presence of the wires, the propagation is enhanced with the signals arriving within $1.58-1.70 \mathrm{~ns}$, which can be seen as strong variation in the impulse responses samples. In addition, Path 6 is clearly noted on the CIRs at the time instant approximately $3 \mathrm{~ns}$ in both cases. Also for the case of the implanted volunteer there are waves arriving slightly earlier due to the propagation path via the wires.
In the impulse responses of the implanted volunteer and nonimplanted volunteer, there is a clear peak with strong variations at the time instant of approximately 2 ns. This is due to Path 4, i.e., propagation through lungs. The strong variation between the samples is due to the respiration, since the propagation time difference between deflated and inflated lungs is remarkable, as can be seen from Table IV. In the presence of the sternotomy wires, the propagation time is $1.33-1.89 \mathrm{~ns}$ depending on the frequency and respiration rate. In general, the variation between the CIR samples is stronger for the implanted case since in this period several waves arrive via different wired paths.

Next, we study the reflection from the aortic valve implant in the case of Path 7. According to the propagation path calculations presented in Section III, the signal reflected from the valve should arrive in the cases of inflated and deflated lungs at times instants 3.22 - $3.47 \mathrm{~ns}$ and 3.70 - $3.88 \mathrm{~ns}$, respectively. When we compare these results with the impulse responses obtained with the implant volunteer and non-implant volunteer shown in Figs 8-9, we can see the differences at these time slots. For the implanted case, we can see peaks and strong variation between the impulse responses on these time instants, since there can be several reflections from the different parts of the valve. Besides, the movements of the valve cause variations on the propagation. On the other hand, the impulse responses of the non-implanted case are very smooth and there is minimal variation between the impulse response samples. As one can note, the channel strongly attenuates the signals at these time instants due to a relatively large distance from the antenna, though some small peaks are seen. Furthermore, it should be noted, that averaged impulse response does not describe the channel characteristics for the implanted case very well since it is relatively flat on these time instants.

For Path 8 (partly through the blood vessels), the signal arrives around $3.4-4$ ns. However, the channel is strongly attenuated at these time instants, especially for the case of dipole antennas.

Finally, these variations are studied with the 3D plots and contour plots presented in Figure 10a for implanted volunteer and in Figure $10 \mathrm{~b}$ for the non-implanted volunteer in more details.

As it can be seen, peak lines of the non-implanted volunteer case are smoother; there is less variation between the CIR samples. With the implanted volunteer, the variation is remarkable, especially in the time slots where impact of the sternotomy wires and the aortic valve implant can be seen ( $2 \mathrm{~ns}, 3 \mathrm{~ns}, 3.5 \mathrm{~ns}, 4 \mathrm{~ns})$. As discussed earlier, the systematic variation between the samples in the time instants that correspond to the impact of the sternotomy wires is explained by the assumption the wire array will store and release energy across time, i.e. like a solenoid being impacted by an EM wave.

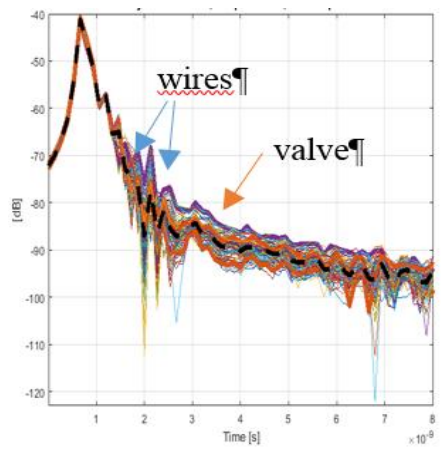

a)

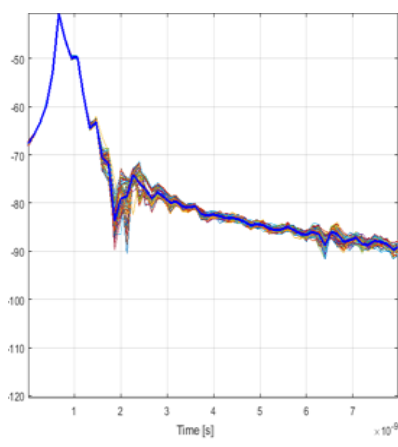

b)
Figure 8. Impulse responses for a) the implanted volunteer and b) the nonimplant volunteer with the loop antenna. 

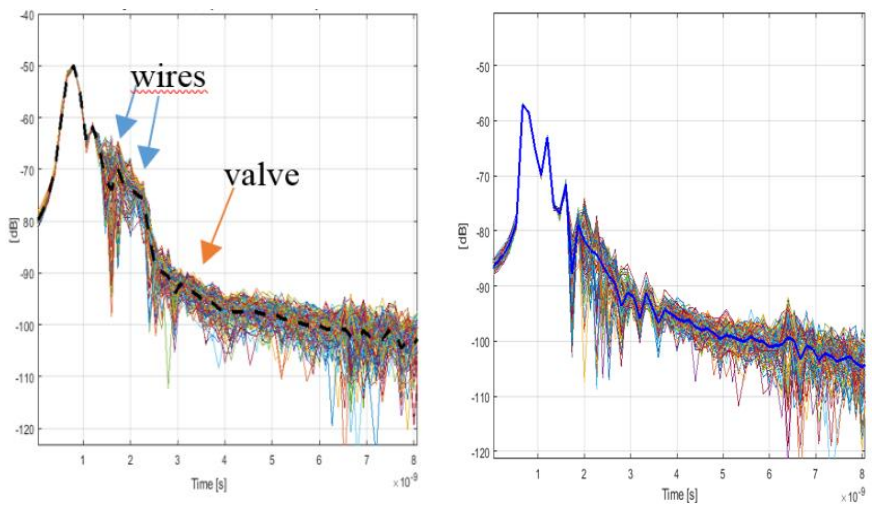

a)

b)

Figure 9. Impulse responses for a) the implanted volunteer and b) nonimplanted volunteer with the dipole antenna.
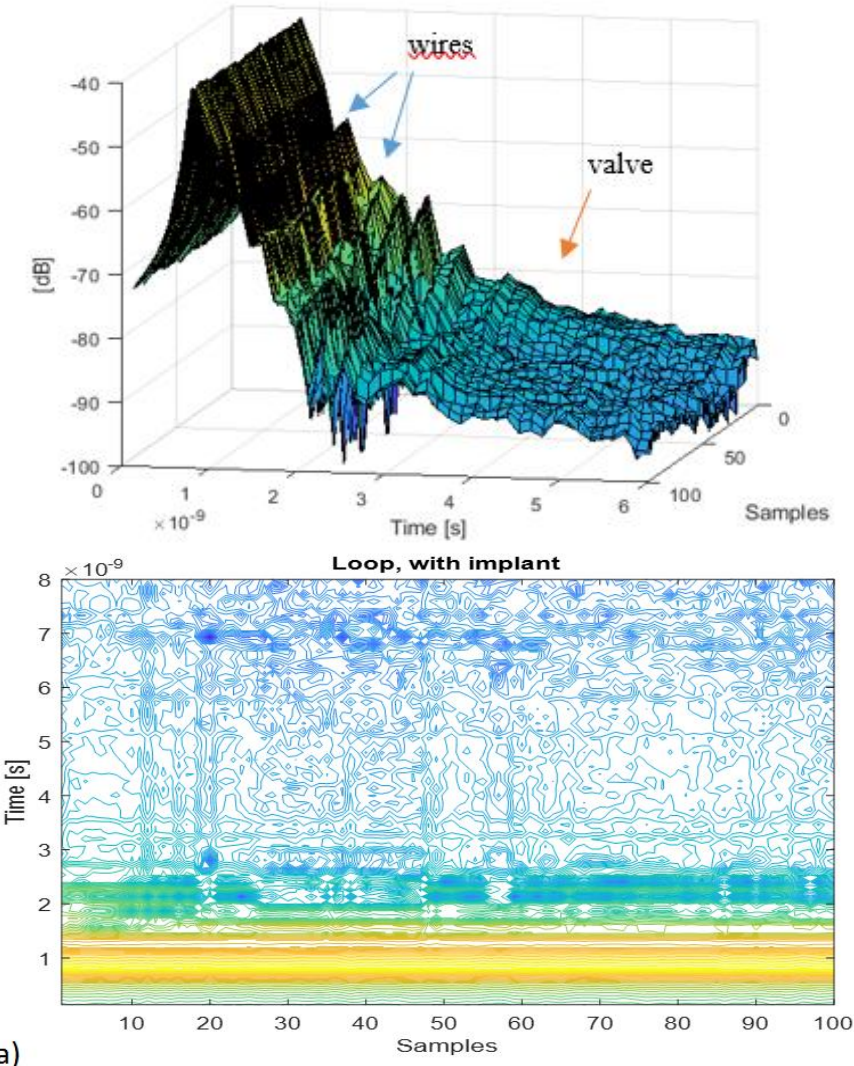

a)

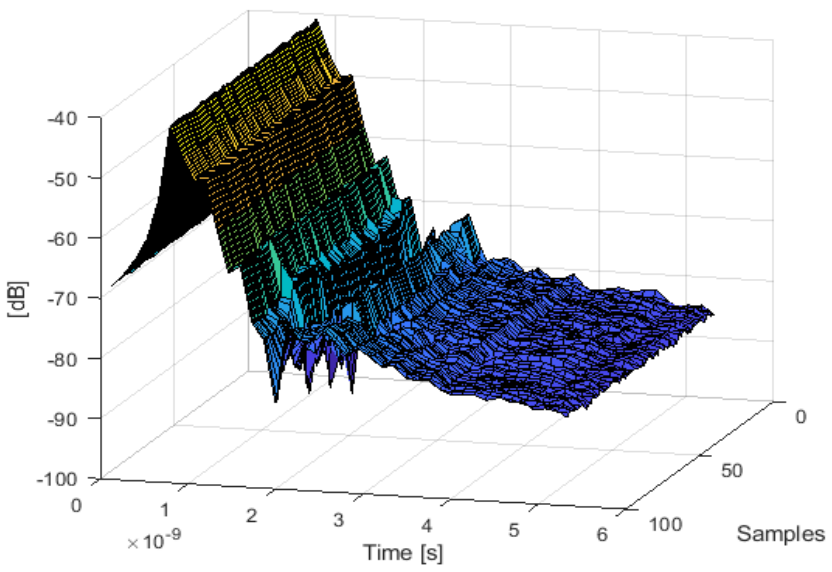

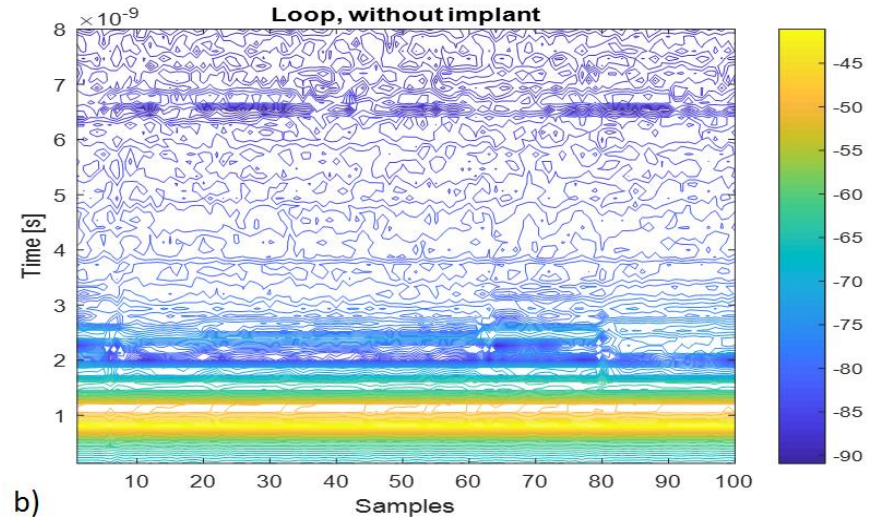

Figure 10. 3D plot and contours the impulse responses obtained with loop antenna a) with implanted volunteer, b) non-implanted volunteer.

When comparing the measurement results obtained with the symmetric antenna layout to the asymmetric antenna layout results in [33], both cases having $15 \mathrm{~cm}$ antenna separation, we can see that the impact of sternotomy wires is stronger in the symmetric case. The variation between the measured samples at the time instant where the impact of sternotomy wires should be seen, is stronger in the symmetric than in the asymmetric case. This is understandable since the signal travels longer way through the wires in the symmetric case. Also the wires above and below the antenna line can have more effect in the symmetric case.

The impact of sternotomy wires and aortic valve implant is clearly visible even with the antennas designed for on- and off-body communications, for which the radiation pattern is preferably outwards from the body. With the antennas designed for intra-body communications, in which the antenna radiation is towards the body, the impact would be stronger.

In the measurement studies, one challenge is the inter-subject variability, which may have strong impact on the differences between the channel responses of different volunteers. For instance, there is a level of differences for the main peaks, shape of the main peak etc. These are commonly known uncertainties in the measurements, as discussed e.g. in [41]. Despite of these inter-subject variabilities, one can note a consistent difference between the implanted and nonimplanted cases at time instants that correspond to propagation path calculations. In order to study pure impact of aortic valve implant and sternotomy wires on the channel characteristics, we conduct simulations with a layer model with and without the implants. The results are presented in Section V.

\section{SIMULATION RESULTS}

Simulation results for the tissue layer model introduced in Section II are presented in this section. The results are verified with numerical calculations and measurement data.

Figures 11 (a) and (b) show the impulse responses obtained for the layer model with a valve implant and sternotomy wires (black solid line) and the reference layer model without any implants (blue dashed line) . Figure 11 (a) is for the loop antenna (a) and Figure 11 (b) is for the dipole antenna. First, we consider the case with the loop antenna. When comparing the impulse responses of implanted and nonimplanted cases, we notice that the main peaks are at the same level but there are changes in the strength and timing of the following side peaks. As explained earlier, the sternotomy wires store and release energy due to their loop form. This phenomenon can be seen at the time instant $0.7 \mathrm{~ns}-1 \mathrm{~ns}$ where the simulated CIR of the nonimplanted layer model is higher than that of the implanted layer 
models. The energy of the loops is released later, for the first time around $1.1 \mathrm{~ns}-1.5 \mathrm{~ns}$. Besides, at the time instant $1.1 \mathrm{~ns}$, appears the additional peak due to the faster propagation in the wires (Path2_w), which will be summed to the energy released from the wire loops. Thus, the level of the CIR is significantly higher at time range $1.1 \mathrm{~ns}$ - $1.2 \mathrm{~ns}$ with the implanted than with the non-implanted case. Next side peak common to both cases arrives at time instant $1.3 \mathrm{~ns}$, which corresponds to propagation path option Path_2 (propagation through skin). One can note that the peak of the implanted layer model is slightly smaller than that of the non-implanted case because part of the signal has propagated through the wires. Paths 4 and 5 both appear around $1.5 \mathrm{~ns}$. As discussed earlier, the impact of the wires below/above antennas can be seen around $1.7 \mathrm{~ns}$ and $2.2 \mathrm{~ns}$, which are clearly visible in the simulated impulse responses. Furthermore, the impact of the aortic valve appears for the first time at $2.4 \mathrm{~ns}$ (Path 6), which is visible in the impulse response as well. The level of the peak is low since the signal reflected from the aortic valve implant is strongly attenuated. In general, the difference between the implanted and non-implanted cases varies between $5 \mathrm{~dB}-17 \mathrm{~dB}$ within the time range corresponding to the relevant propagation paths.

Similar tendency can be found in the impulse responses obtained using a dipole antenna as shown in Figure 11 (b). The impact of the sternotomy wires and the aortic valve implant can be seen in the same time ranges as with the loop antenna. However, the impact of the sternotomy wires is clearest around $2 \mathrm{~ns}$. Besides, the impact of aortic valve implant is weaker than with the loop antenna. This is assumed to be due to different radiation patterns close to the human body [35] Next, the propagation path options are validated by calculating the power loss for each path. The calculation is based an extension of the algorithm that includes lossy media proposed by Orfanides [40]. Due to lack of the space, the power losses for each path is only summarized in Table V. One should note that power loss is calculated for each path option separately, i.e., all the transmitted power is directed to the evaluated path option. In this way, different propagation path options are compared equally. However, in the measurements or in the CST simulations, the transmitted energy is spread for all the directions taking into account the antenna radiation pattern at each frequency. Thus, these power loss results cannot be compared directly with the peak levels of the simulated and measured data.
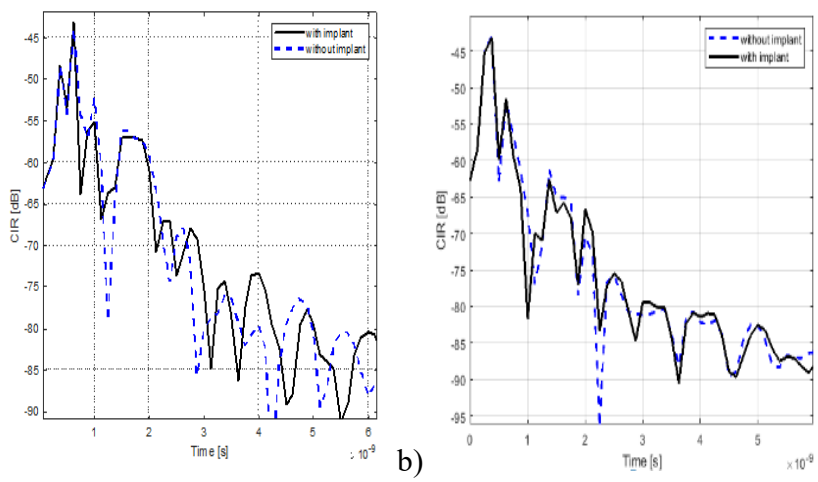

b)

Figure 11 (a) and (b). Channel impulse responses for the implanted case and non-implanted case using a) loop antenna and b) dipole antenna.

As one can see from Table $\mathrm{V}$, power loss for different path options varies depending on the tissues of the path option as well as on the frequency. For most of the path options, the power loss is close to 30 $\mathrm{dB}$ at $2.5 \mathrm{GHz}$ and around $100 \mathrm{~dB}$ at $8 \mathrm{GHz}$. Propagation through the fat layer makes an exception: the power loss is only $12 \mathrm{~dB}$ at $2.5 \mathrm{GHz}$ and only $38 \mathrm{~dB}$ at $8 \mathrm{GHz}$. As a comparison, the power loss for skin at $8 \mathrm{GHz}$ is $117 \mathrm{~dB}$. Thus, most of the signal arriving to $\mathrm{Rx}$ through the tissues is from the lower frequency band. Fat layer seems to be the only possible propagation path option at higher frequencies.

Table V Power loss for each of the path option in [dB]

\begin{tabular}{|l|l|l|l|l|}
\hline Path option / frequency & $2.5 \mathrm{GHz}$ & $3.5 \mathrm{GHz}$ & $5 \mathrm{GHz}$ & $8 \mathrm{GHz}$ \\
\hline Path 1 & 31.91 & 41.45 & 60.12 & 111.74 \\
\hline Path1w & 26.02 & 33.35 & 47.69 & 87.35 \\
\hline Path 2 & 30.27 & 36.74 & 56.83 & 100.78 \\
\hline Path 2_w & 26.15 & 36.68 & 54.80 & 95.92 \\
\hline Path 3 & 12.68 & 17.34 & 24.41 & 37.57 \\
\hline Path3_w & 12.37 & 16.66 & 22.19 & 32.53 \\
\hline Path 4 & 27.55 & 43.94 & 68.62 & 115.49 \\
\hline Path 4w & 26.59 & 42.62 & 68.11 & 117.13 \\
\hline Path 5 & 29.18 & 63.54 & 97.03 & 176.71 \\
\hline Path5w & 23.94 & 62.19 & 98.43 & 174.94 \\
\hline Path 6 & 68.64 & 96.81 & 147.06 & 269.38 \\
\hline Path 6_w & 63.63 & 95.17 & 102.57 & 193.49 \\
\hline
\end{tabular}

\section{CONCLUSIONS}

In this paper, we have presented a study on the impact of sternotomy wires and aortic valve implant on the radio channel characteristics, within the human body, by studying intra-body propagations in the vicinity of on-body antennas. Propagation path calculations are compared with measurement data obtained from a volunteer that has sternotomy wires and an aortic valve implant as well as reference volunteers without any implants. Furthermore, the impact is studied using a tissue layer models designed according to the dimensions of the implanted volunteer.

The correspondence between the propagation path calculations and measurement data is clear; there are peaks in the radio channel impulse response at the epochs that correspond to the calculated arrival times of propagation paths. Sternotomy wires and the aortic valve implant cause additional peaks in the channel impulse response as well as stronger variation between consecutive channels. Additionally, the simulation results obtained with a tissue layer model with and without wires and implant clearly emphasize the impact of the sternotomy wires and aortic valve implant on the channel impulse response. The strongest impact is due to the sternotomy wires, which are close to the skin surface and hence close to the antennas. The impact of the aortic valve implant is weaker since the valve is located deep inside the body (approximately $7 \mathrm{~cm}$ from the body surface for an average size adult) and the signal reflected from the valve, is strongly attenuated. However, one should note that in this study case we have used antennas designed for on- and off-body communication. With antennas designed for intra-body communications, the impact of the sternotomy wires and aortic valve implant will be even more significant.

Knowledge about the impact of the sternotomy wires and aortic valve implant is important and should be taking into account when designing on-body monitoring devices. Especially the location of the Tx and Rx antennas should be planned carefully to avoid multipath interference. The closer the Tx and Rx antennas are to each other, the stronger is the impact. Particularly the sternotomy wires could cause interfering additional peaks on the impulse response since they are located close to the skin. As discussed in the Section IV, the impact of the sternotomy wires is more significant if the antennas are located symmetrically respect to the sternum. Our future target is to study in more detail the impact of medical wires and implants on the channel characteristics with different antenna locations and antenna types. Furthermore, one of our aims is to find antenna locations in which the valve implant has the strongest impact on the channel characteristics. 
Our assumption is that with appropriate antenna locations and highly directive on-body antennas designed for intra-body communication, even the valve movements could be detected during the heartbeat period. This knowledge may be utilized in the design of a monitoring device observing the functionality of the valve.

\section{ACKNOWLEDGMENT}

This research has been financially supported by the project WBAN Communications in the Congested Environments and in part by Academy of Finland 6Genesis Flagship (grant 318927).

We thank the volunteers who participated in the measurements. Jari Sillanpää, Ilkka Virtanen, and Timo Mäkinen from University of Oulu are acknowledgement for their help to enable the simulations.

\section{REFERENCES}

[1] A. F. Demir, Q. H. Abbasi, Z. E. Ankarali, A Alomainy, K.Qaraqe, E. Serpedin, H. Arsalan, "Anatomical Region-Specific In Vivo Wireless Communication Channel Characterization," IEEE Journal on Biomedical and Health Informatics, 2017.

[2] A. F. Demir et al., "In vivo communications: Steps toward the next generation of implantable devices", IEEE Vehicular Technology Magazine, 2016.

[3] R. Chávez-Santiago et al., "Experimental path loss models for in-body communications within 2.36-2.5 GHz", IEEE Journal on Biomedical Health Informatics, 2015.

[4] P. Turalchuk, I. Munina, V. Pleskachev, V. Kirillov, O. Vendik, I. Vendik, "In-Body and On-Body Wave Propagation: Modeling and Measurements," International Workshop on Antenna Technology: Small Antennas, Innovative Structures, and Applicattions (iWAT), 2017.

[5] N. B. Asan, E. Hassan, JVSRM Shah, D. Noerland, T.J. Blokhuis, E. Wadbro, M. Berggren, T. Voig, R. Augustine, "Characterization of the Fat Channel for Intra-Body Communication at R-Band Frequencies," MDPI Sensors 2018, 2018.

[6] N. R. Amon, I. Mahbub, P: Kanai. Saha, "Propagation Characteristics of Ultra-Wideband Pulse in Multilayered Human Chest Tissue," International Conference on Electrical Engineering and Information Communication Technology (ICEEICT), 2016.

[7] I. Dove, "Analysis of Radio Propagation Inside the Human Body for InBody Localization Purposes", Master Thesis, Faculty of Electrical Engineering, Mathematics and Computer Science, University of Twente, Netherland, 2014.

[8] Y. El-Saboni, G. A. Conway, S. L. Cotton, W. G. Scanlon, "Radiowave Propagation Characteristics in the Intra_Body Channel at $2.38 \mathrm{GHz}$ ", IEEE International Conference on Wearable and Implantable Body Sensor Networks (BSN), 2017.

[9] K. N. Sahu, C. D. Naidu, M. Satyam, K. Jaya Sankar, "Study of RF Signal Attenuation of Human Heart," Hindawi Journal on Engineering, 2015.

[10] S. N. Makarov, G. M. Noetscher, J. Yanamadala, M. W. Piazza, S. Louie, A. Prokop, A. Nazarian, A. Nummenmaa, "Virtual Human Models for Electromagnetic Studies and Their Applications," IEEE Review in BioMedical Engineering, 2017.

[11] J. Li, Z. Nie, Y. Liu, L. Wang, Y. Hao, "Characterization of In-Body Radio Channels for Wireless Implants," IEEE Sensors Journal, 2017.

[12] A. Khaleghi, R. Chavez-Santiago, I. Balasingham, "Ulta-wideband statistical propagation channel model for implant sensors in the human chest," IET Microwaves, Antennas \& Propagation, 2011.

[13] A. Alomainy, Y. Hao, "Modeling and Characterization of Biotelemetric Radio Channel From Ingested Implants Considering Organ Contents," IEEE Transaction on Antennas and Propagation, 2009.

[14] A. Sani, A. Alomainy, Y. Hao, "Numerical Characterization and Link Budget Evaluation of Wireless Implants Considering Different Digital Human Dhantame", IEEE Transactions on Microwave Theory and Techniques, 2009.

[15] C. Garcia-Pardo, A. Fornes-Leal, N. Cardona, S. Brovoll, o. Aardal, S.E. Hamran, R. Chavez-Santiago, J. Berland, I. Balasingham, R. Palomar, "Experimental Ultra Wideband Path Loss Model for Implant Communication," IEEE International Symposium on Personal Indoor and Mobile Radio Communications (PIMRC), 2016.
[16] A. K. Teshome, B. Kibret, D.T. H. Lai, "Galvinically Coupled Intrabody Communications for Medical Implants: Unified Analytic Model," IEEE Transaction on Antennas and Propagation, 2016.

[17] P. T. Theilmann, M. A. Tassoudji, E. H. Teague, D. F. Kimball, P. M. Asbeck, "Computationally Efficient Model for UWB Signal Attenuation due to Propagation in Tissue for Biomedical Implants," Progress in Electromagnetics Research B, 2012.

[18] P. A. Floor, R. Chavez-Santiago, S. Brovoll, O. Aardal, J. Bergsland, OJ. H. N. Grymyr, P. Halvorsen, R. Palomar, D. Plettemeier, S-E. Hamran, T. Ramstad, I. Balasingham, "In-Body to On-Body Ultrawideband Propagation Model Derived from Measurement in Living Animals," IEEE Journal of Biomedical and Health Informatics, 2015.

[19] A. Daisuke, K. Katsu, R. Chávez-Santiago, Q. Wang, D. Plettemeier, J. Wang, I. Balasingham, "Experimental evaluation of implant UWB-IR transmission with living animal for body area networks", IEEE Transaction on Microwave Theory Technology, 2014.

[20] X. Yang, M. Fang, A. Ren, Z. Zhang, Q. Abbasi, A. Alomainy, K. Mehran, "Reverse Recognition of Body Postures using On-Body Radio Channel Characteristics," IET Microwaves, Ant., and Prog, 2017.

[21] T. Kumpuniemi, M. Hämäläinen, K. Y. Yazdandoost, J. Iinatti, "Human Body Shadowing Effect on Dynamic UWB On-Body Radio Channels," IEEE Antennas and Wireless Propagation Letters, 2017.

[22] T. Kumpuniemi, M. Hämäläinen, K. Y. Yazdandoost, J. Iinatti, "Categorized UWB On-Body Radio Channel Modeling for WBANs," Progress in Electromagnetic Research B, 2016.

[23] A. Taparugssanagorn, C. Pomalaza-Ráez, A. Isola, R. Tesi,M. Hämäläinen, J. Iinatti, "UWB Channel Modeling for Wireless Body Area Networks in Medical Applications," Applied Sciences in Biomedical and Communication Technologies (ISABEL), 2009.

[24] A. Taparugssanagorn, C. Pomalaza-Ráez, A. Isola, R. Tesi,M. Hämäläinen, J. Iinatti, "Preliminary UWB Channel Study for Wireless Body Area Networks in Medical Applications" International Journal of UWB and Systems, InderScience Publishers, 2011.

[25] W.-B. Yang, K. Sayrafian-Pour, J. Hagedorn, J. Terrill, K.Y. Yazdandoost, A. Taparugssanagorn, M. Hämäläinen, J. Iinatti, "Impact of an Aortic Valve Implant on Body Surface UWB Propagation: A Preliminary Study", Proc. the 5th International Symposium on Medical Information and Communication Technology, 2011.

[26] M. Särestöniemi, M. Hämäläinen, J. Iinatti, "An Overview of Electromagnetic Propagation Based Channel Modeling Techniques for Wireless Body Area Network Applications," IEEE Access, 2017.

[27] A. Pellegrini, A. Brizzi, L. Zhang, K. Ali, Y. Hao, X. Wu, C. C. Constantinou, Y. Nechayev, P. S. Hall, N. Chahat, M. Zhadobov, R. Sauleau, "Antennas and Propagation for Body-Centric Wireless Communications at Millimeter-Wave Frequencies: A Review [Wireless Corner]", Antennas and Propagation Magazine IEEE, 2013.

[28] K. M. S. Thotahewa, J-M. Redoute, M. R. Yuce, "Propagation, Power Absorption, and Temperature Analysis of UWB Wireless capusle Endoscopy Devices Operating in the Human Body," IEEE Transactions on Microwave Theory, 2015.

[29] M. Amparo Callejon, Javier Reina-Tosina, David Naranjo-Hernandez, Laura M. Roa, "Galvanic Coupling Transmission in Intrabody Communication: A Finite Element Approach", IEEE Transactions on Biomedical Engineering, vol. 61, pp. 775-783, 2014.

[30] S. Mohan, A. Boddupulli, Md. Delwar Hossain, F. Gozasht, S. S. H. Ling, "Techniques for RF Localization of Wireless Capsule Endoscopy," International Conference on Electromagnetics in Advanced Applications (ICEAA), 2016.

[31] A.Elfström, A. Grunditz, "Evaluation of Sternum Closure Techniques Using Finite Element Analysis, " Master Thesis, in Medical Engineering, The Royal Institute of Technology, Sweden, 2013.

[32] M. Särestöniemi, T. Kumpuniemi, M. Hämäläinen, C. Pomalaza-Raez, J. Iinatti, "Impact of the Sternotomy Wires and Aortic Valve Implant on the On-body UWB Radio Channel," International Symposium on Medical Information and Communication Technology (ISMICT2018), 2018.

[33] M. Särestöniemi, T. Kumpuniemi, M. Hämäläinen, C. Pomalaza-Raez, J. Iinatti, "Finite Integration Technique Based Simulation Study on the Impact of Sternotomy Wires on the UWB Channel Characteristics, International Symposium on Wireless Body Area Networks (BodyNets2018), 2018. 
[34] Medtronic aortic valve, URL:http://www.15tronic.com/us-en/healthcareprofessionals/products/cardiovascular/heart-valves-surgical.html

[35] T. Tuovinen, K. Yekeh Yazdandoost, J. Iinatti, "Comparison of the Performance of Two Different UWB Antennas for the use in WBAN OnBody Coimmunications," European Conference on Antennas and Propagation (EUCAP2012), 2012.

[36] SkyCross Antenna Product, www.skycross.com

[37] T. Tuovinen, T. Kumpuniemi, K. Y. Yazdandoost, M. Hämäläinen, J. Iinatti, "Effect of the Antenna-Human Body Distance on the Antenna Matching in UWB WBAN Applications," ISMICT2013, 2013.

[38] CST Microwave Studio, [Online]. Available: http://www.cst.com.

[39] https://www.itis.ethz.ch/virtual-population/tissueproperties/database/dielectric-properties.

[40] S. J. Orfanidis, "Electromagnetic Waves and Antennas," 2002, revised 2016, online: http://www.ece.rutgers.edu/ orfanidi/ewa/.

[41] T. Kumpuniemi, T. Tuovinen, M. Hämäläinen, K. Yekeh Yazdandoost, R. Vuohtoniemi, J. Iinatti, "Measurement-Based On-Body Path Loss Modelling for UWB WBAN Communications", ISMICT2013, 2013.

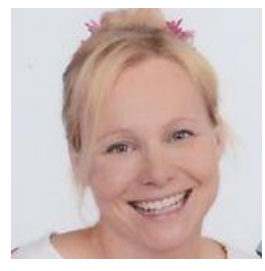

Mariella Särestöniemi received her M. Sc. and Lic. Tech degrees in 2003 and 2005, respectively, from University of Oulu, Finland. Currently, she is a doctoral student at Centre for Wireless Communications at the University of Oulu. Her research interests are medical ICT, wireless body area networks, in-body and on-body communications, simulation based channel modeling and measurements.

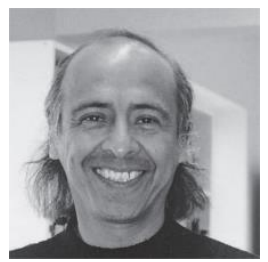

Carlos Pomalaza-Ráez (M'80-SM'06) received the B.S.M.E. and B.S.E.E. degrees from Universidad Nacional de Ingeniería, Lima, Peru, in 1974, and the M.S. and Ph.D. degrees in electrical engineering from Purdue University, West Lafayette, IN, USA, in 1977 and 1980, respectively. He is an electrical engineering Professor at Purdue University, Fort Wayne, Indiana, USA. He has been a faculty member at the University of Limerick, Limerick, Ireland, and at Clarkson University, Potsdam, NY, USA. He has also been a Member of the Technical Staff at the Jet Propulsion Laboratory, California Institute of Technology, Pasadena, CA, USA. In 2003-2004, under the auspices of a Nokia-Fulbright Scholar Award, he was a Visiting Professor at the Centre of Wireless Communications, University of Oulu, Oulu, Finland. His research interests include wireless communications networks and signal processing applications.

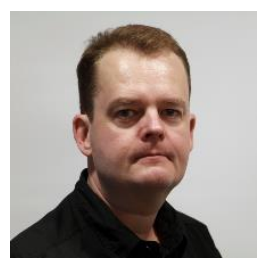

Timo Kumpuniemi received his M. Sc.(Tech.) and Lic. Sc. (Tech.) degrees in 2001 and 2008, respectively, from University of Oulu, Finland. He is at the moment working as a doctoral student at Centre for Wireless Communications at the University of Oulu. His current research interests cover wireless medical communications, biomedical engineering, wireless body area networks, visible light communications, radio engineering on various aspects and modulation methods, including spread spectrum and ultra wideband systems. His teaching activities have been mainly on different fields of radio engineering, including also other fields of telecommunications engineering.

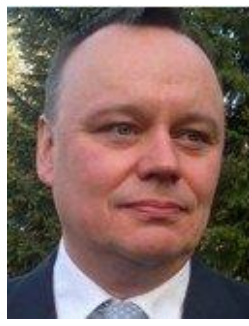

Matti Hämäläinen (SM'09) received his M.Sc, Lic.Tech. and Dr.Sc. degrees in 1994, 2002 and 2006, respectively, from University of Oulu, Finland. Currently he is Adjunct Professor and University Researcher at Centre for Wireless Communications, University of Oulu, Finland and IAS Visiting Professor at Yokohama National University, Japan. His research interests are in ultra wideband systems, radio channel modeling, wireless body area networks and medical ICT.

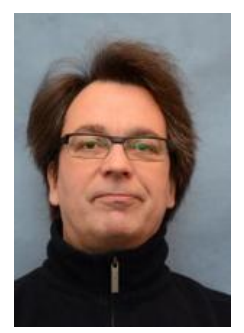

Jari Iinatti (SM'05) received M.Sc., Lic.Tech., and Dr.Tech. degrees in electrical engineering from the University of Oulu, Oulu, Finland, in 1989, 1993, and 1997, respectively. From 1989 to 1997, he was a Research Scientist with the Telecommunication Laboratory, University of Oulu. From 1997 to 2002, he was acting Professor of Digital Transmission Techniques, and a Senior Research Scientist, Project Manager, and Research Director with the Center for Wireless Communications, University of Oulu. Since 2002, he has been a Professor of Telecommunication Theory. Currently he is head of Centre for Wireless Communcations Networks and Systems. His research interests include future wireless communications systems, transceiver algorithms, wireless body area networks (WBANs), and medical ICT. He has authored over 200 international journal and conference papers, holds six patents, and is a co-editor of the book UWB Theory and Applications (Wiley \& Sons, Ltd., Chichester, U.K., 2004). He has supervised 13 doctoral theses and over 60 master's theses. He has been a Technical Program Committee (TPC) member in about 25 conferences, and he was a TPC Co-Chair in the IEEE PIMRC2006, BodyNets2012 and PIMRC2014, a TPC chair in the ISMICT2007, a General Co-Chair in the ISMICT2011, ISMICT2014 and ISMICT2015, and a TPC Program Track CoChair in BodyNets 2012. He was also an organizer of the FEELIT 2008, the FEELIT2011, the UWBAN2012, and the UWBAN2013. He is Steering Committee Co-Chair of ISMICT series. 